\title{
Reported barriers to evaluation in chronic care: Experiences in six European countries
}

Cécile Knai $^{\mathrm{a}, *}$, Ellen Nolte ${ }^{\mathrm{b}}$, Matthias Brunn ${ }^{\mathrm{c}}$, Arianne Elissen ${ }^{\mathrm{d}}$, Annalijn Conklin ${ }^{\mathrm{b}, \mathrm{e}}$, Janice Pedersen Pedersen ${ }^{\mathrm{b}}$, Laura Brereton ${ }^{\mathrm{b}}$, Antje Erler ${ }^{\mathrm{f}}$, Anne Frølich $^{\mathrm{g}}$, Maria Flamm ${ }^{\mathrm{h}, \mathrm{n}}$, Birgitte Fullerton ${ }^{\mathrm{f}}$, Ramune Jacobsen ${ }^{\mathrm{g}}$, Robert Krohn $^{\mathrm{i}}$, Zuleika Saz-Parkinson ${ }^{\mathrm{j}}$, Bert Vrijhoef ${ }^{\mathrm{k}}$, Karine Chevreul ${ }^{\mathrm{c}, 1}$, Isabelle Durand-Zaleski ${ }^{c, 1}$, Fadila Farsi ${ }^{m}$, Antonio Sarría-Santamera ${ }^{\mathrm{j}}$, Andreas Soennichsen ${ }^{\mathrm{n}}$

\footnotetext{
a London School of Hygiene E' Tropical Medicine, Faculty of Public Health and Policy, 15-17 Tavistock Place, London WC1H9SH, United Kingdom

b RAND Europe, Health and Healthcare Policy, Westbrook Centre, Milton Road, Cambridge CB4 1YG, United Kingdom

c URC Eco, UPEC/AP-HP, 228 rue du Fg St Martin, 75010 Paris, France

d Maastricht University, Duboisdomein 30, 6229 GT Maastricht, The Netherlands

e Centre for Diet and Activity Research, University of Cambridge, CB2 OSR, United Kingdom

f Johann Wolfgang Goethe University, Theodor-Stern-Kai 7, D-60590 Frankfurt, Germany

$\mathrm{g}$ University of Copenhagen, Jagtvej 160, 2100 Copenhagen, Denmark

h Danube University, Department of Evidence-based Medicine and Clinical Epidemiology, Danube University, Karl Dorrek-Straße 30,

3500, Krems, Austria

i AQUA-Institut für angewandte Qualitätsförderung und Forschung im Gesundheitswesen GmbH, Maschmühlenweg 8-10, 37073

Göttingen, Germany

j Instituto de Salud Carlos III, Avda. Monforte de Lemos, 5, 28029 Madrid, Spain

k Scientific Center for Care and Welfare (TRANZO), Tilburg University, PO Box 90153, 5000 LE Tilburg, The Netherlands

${ }^{1}$ AP-HP Recherche Clinique Santé Publique, Hôpital Henri Mondor, France

m Réseau Espace Santé Cancer Rhône-Alpes, France

n Institute of General Practice, Paracelsus Medical University, Strubergasse 21, 5020 Salzburg, Austria
}

\section{A R T I C L E I N F O}

\section{Article history:}

Received 16 May 2012

Received in revised form 7 November 2012

Accepted 17 January 2013

\section{Keywords:}

Evaluation

Chronic care

Europe

Chronic disease

Barriers

\begin{abstract}
A B S T R A C T
Introduction: The growing movement of innovative approaches to chronic disease management in Europe has not been matched by a corresponding effort to evaluate them. This paper discusses challenges to evaluation of chronic disease management as reported by experts in six European countries.

Methods: We conducted 42 semi-structured interviews with key informants from Austria, Denmark, France, Germany, The Netherlands and Spain involved in decision-making and implementation of chronic disease management approaches. Interviews were complemented by a survey on approaches to chronic disease management in each country. Finally two project teams (France and the Netherlands) conducted in-depth case studies on various aspects of chronic care evaluation.
\end{abstract}

\footnotetext{
* Corresponding author at: European Health Policy, London School of Hygiene and Tropical Medicine, 15-17 Tavistock Place, London WC1H9SH, United Kingdom. Tel.: +440207958 8155 .

E-mail addresses: cecile.knai@lshtm.ac.uk(C. Knai), enolte@rand.org(E. Nolte), matthias.brunn@urc-eco.fr(M.Brunn), a.elissen@maastrichtuniversity.n (A. Elissen), annalijn.conklin@mrc-epid.cam.ac.uk (A. Conklin), pedersen@rand.org (J.P. Pedersen), brereton@rand.org (L. Brereton), erler@allgemeinmedizin.uni-frankfurt.de (A. Erler), anne.frolich@dadlnet.dk (A. Frølich), Maria.Flamm@donau-uni.ac.at (M. Flamm), Fullerton@allgemeinmedizin.uni-frankfurt.de (B. Fullerton), raja@farma.ku.dk (R. Jacobsen), robert.krohn@aqua-institut.de (R. Krohn), zuleika@isciii.es (Z. Saz-Parkinson), h.j.m.vrijhoef@uvt.nl (B. Vrijhoef), karine.chevreul@hmn.aphp.fr (K. Chevreul), isabelle.durand-zaleski@hmn.ap-hop-paris.fr (I. Durand-Zaleski), fadila.farsi@rrc-ra.fr (F. Farsi), asarria@isciii.es (A. Sarría-Santamera), andreas.soennichsen@pmu.ac.at (A. Soennichsen).
} 
We identified three common challenges to evaluation of chronic disease management approaches: (1) a lack of evaluation culture and related shortage of capacity; (2) reluctance of payers or providers to engage in evaluation and (3) practical challenges around data and the heterogeity of IT infrastructure. The ability to evaluate chronic disease management interventions is influenced by contextual and cultural factors.

Conclusions: This study contributes to our understanding of some of the most common underlying barriers to chronic care evaluation by highlighting the views and experiences of stakeholders and experts in six European countries. Overcoming the cultural, political and structural barriers to evaluation should be driven by payers and providers, for example by building in incentives such as feedback on performance, aligning financial incentives with programme objectives, collectively participating in designing an appropriate framework for evaluation, and making data use and accessibility consistent with data protection policies. (c) 2013 Elsevier Ireland Ltd. All rights reserved.

\section{Introduction}

European countries are experimenting with a range of innovative approaches to better manage chronic disease [1-4]. Several have opted for structured disease management as a means to improve the quality and, potentially, reduce the cost of healthcare, and to improve health outcomes for those with chronic conditions. While intuitively appealing, the evidence that such approaches can in fact achieve these goals remains uncertain. Current evidence is largely based on small studies of high-risk patients, often undertaken in academic settings while systematic, scientifically robust evaluations of larger scale interventions and approaches remain scarce [5-7]. There is a comparatively large knowledge base on the methodological and practical challenges to evaluating disease management interventions [8-10] and more generally, complex interventions in healthcare [5,11-15]. The context for evaluation has been less extensively discussed, typically focusing on the setting within which evaluation takes place vis-à-vis the nature of the intervention to be evaluated [12]. There has been limited attention on unravelling the cultural, political and technological factors that may hinder systematic evaluation of complex healthcare interventions. Understanding these barriers is essential to encourage evaluation and in turn, generate evidence-based decision-making in chronic care.

This paper aims to contribute a better understanding of barriers to evaluation in chronic care. We examine in particular the broader perspective of key stakeholders on the evaluation of chronic disease management approaches as part of an overall assessment of health system performance.

\section{Methods}

This paper builds on work conducted by the European DISMEVAL project (Developing and validating DISease Management EVALuation methods for European healthcare systems), which sought to review current approaches to chronic care and their evaluations in EU Member States and to test and validate methods and metrics for their evaluation. We report on data collected from key informant interviews and a survey of chronic disease management approaches in the six partner countries, which informed this work. We complement these data with two in-depth case studies in France and The Netherlands.

\subsection{Key informant interviews}

We carried out semi-structured interviews with key informants from Austria, Denmark, France, Germany, The Netherlands, and Spain involved in the decision-making process as it relates to various aspects of chronic disease management in a given health system context.

The six countries were selected to capture the range of approaches to funding and governing healthcare across Europe: all six have a similar commitment to providing universal and reasonably equitable access to healthcare for their populations, but do so in different ways. Denmark and Spain operate primarily tax-funded systems, and the health systems in Austria, France, Germany and the Netherlands are primarily funded through statutory health insurance. Countries also represent different governance systems. France is characterised by structures that tend to be concentrated at the central (national) level, with decentralisation of some functions to regional agencies. In Denmark and Spain, administrative and political responsibility is partly or fully devolved to local or regional authorities; in Austria and Germany, it is devolved to state governments. Moreover, in Austria, Germany and The Netherlands, corporate actors (for example health insurance, providers) play an important role in healthcare governance [16]. It is our hypothesis that contextual, cultural, organisational and other features of the health system will influence the way chronic care initiatives are being implemented and, by extension, whether/how they are being evaluated.

We approached individuals in senior positions representing the decision-maker, payer, provider and/or patient perspective (Table 1). Study participants were identified through purposive and 'snowball' sampling, drawing from an established professional network of international contacts and through project partners based in the six countries.

Three researchers were present during each interview; one led the discussion (either CK or EN), a second person listened for key areas to explore further, and a third person took notes. Interviews were undertaken as telephone interviews between July and October 2010, using a semistructured interview guide. The majority of interviews were held in English, with native language explanations 
Table 1

Number of interviews by sector and country.

\begin{tabular}{|c|c|c|c|c|c|c|c|}
\hline \multirow[t]{2}{*}{ Sectors } & \multicolumn{7}{|c|}{ Countries } \\
\hline & AUS & DEN & FRA & GER & NET & SPA & Total \\
\hline $\begin{array}{l}\text { Decision-maker (ministry of health, government agencies, } \\
\text { local government, regulators) }\end{array}$ & 2 & 2 & 1 & 2 & 4 & 3 & 14 \\
\hline Payer (sickness funds, health insurers) & 2 & - & - & 1 & 2 & - & 5 \\
\hline Provider (GPs, medical associations, hospital associations) & 1 & 1 & - & 2 & 1 & - & 5 \\
\hline Patient (patient associations) & - & - & 1 & - & - & 1 & 2 \\
\hline $\begin{array}{l}\text { Other (disease associations, researchers, health educators, } \\
\text { independent councils for chronic disease/health } \\
\text { care/public health) }\end{array}$ & - & 4 & 1 & 3 & 4 & 4 & 16 \\
\hline Total & 5 & 7 & 3 & 8 & 11 & 8 & 42 \\
\hline
\end{tabular}

AUS: Austria; DEN: Denmark; FRA: France; GER: Germany; NET: Netherlands; SPA: Spain.

where necessary and appropriate, and lasted one hour on average. One interview was held entirely in French and one in Spanish. Two study participants declined to be interviewed in person but provided their answers in writing.

The interview topics included: (1) participants' views on the main focus, along the care continuum, of a country's chronic care efforts; (2) perceived successes and failures in chronic care in their country; and (3) perceived barriers to developing, implementing, and evaluating chronic care approaches. All interviews were recorded and transcribed following consent.

A range of frameworks and theories have been developed to analyse policy and its many components such as agenda setting, policy development, and policy change [17-19]. The conceptual framework underpinning this study is the 'policy triangle framework' $[20,21]$, an influential framework developed partly in response to the observation that health policy research tended to focus on the content of policy rather than the actors, context and processes and their relationships which play an important role in policy development and implementation [22]. Influencing these interlinked components is a range of external or environmental factors, including political, administrative, economic and other considerations. There are several ways of categorising these factors purposely, and we drew upon Leichter's categories of factors, namely situational, structural, environmental and cultural, to guide our analysis [23].

Findings were processed and analysed using the principles of framework analysis, considered appropriate as it is explicitly relevant to applied policy research [24]. A preliminary list of key ideas and recurrent themes were identified from a careful reading of the interview transcripts and then recorded in a purposely built matrix. Data were then gradually organised into categories, facilitating the description of the data, interconnections between the data, and eventually the generation of explanatory patterns.

The London School of Hygiene \& Tropical Medicine Ethics Committee granted ethical approval for the study.

\subsection{Survey of chronic disease management evaluation approaches}

Interviews were complemented by data collected by project partners using a common questionnaire which is described in detail elsewhere [16]. The approach to the survey data collection has also been described in detail elsewhere [7]. In brief, we compiled information on approaches to chronic disease management in European healthcare systems and, as part of this, also collected data on methods and metrics used to evaluate these approaches. This included, for example, evaluation goals and design, indicators of programme effect, data sources, and use of evaluation findings to inform policy, alongside reported and potential barriers to evaluating chronic care initiatives. Principal data collection was carried out from June to December 2009. Each completed template formed the basis for a country report, which was reviewed by the key informant leading template completion for each country to ensure accuracy and allow for the update of information where necessary and appropriate, during summer 2011.

\subsection{Case studies}

Two case studies developed by the French and Dutch DISMEVAL partners, respectively provide additional insights into the broader context for evaluation and challenges encountered. The first examined the existing statutory evaluation requirements of provider networks in France to inform current evaluation practice. The analyses undertaken sought to better understand how, within this broad regulatory environment, provider networks approach evaluation [25]. In particular it aimed to assess the link between objectives and evaluation methods, the appropriateness of the evaluation approach, and the adherence to national recommendations. The analysis drew on data provided by 12 provider networks addressing common conditions such as diabetes, but also less common conditions such as multiple sclerosis. Analyses included non-public external evaluations of the most recent triennial period as well as internal activity reports (2007-2009). In addition, 13 semi-structured interviews were conducted with provider network coordinators, evaluators, stakeholders and funding entities [25].

The second case study collected qualitative data on information needs and measures of success by Dutch practitioners involved in integrated service delivery for specific chronic conditions - i.e. type 2 diabetes, chronic obstructive pulmonary disease (COPD) and vascular risks which are covered under the bundled payment system for 
long-term care. Interviews were conducted with a selection of 27 healthcare professionals from 9 diabetes care groups (a manager, a GP, and a practice nurse per group) covering four broad topics: (1) determining and measuring the goals of diabetes care; (2) function and possibilities of evaluation; (3) type and range of quality indicators; and (4) indicator development.

This paper reports on the survey and interview findings pertaining to the challenges to evaluation, supplemented by the results of the two case studies.

\section{Results}

We identified three common challenges to evaluation: (1) a lack of evaluation culture and related shortage of capacity; (2) reluctance of payers or providers to engage in evaluation and (3) practical challenges around data and the heterogeity of IT infrastructure. We explore each of these themes in turn.

\subsection{Perceived lack of evaluation culture}

Survey reports demonstrated a 'lack of a general evaluation culture' as one main barrier towards producing sound evidence on disease management approaches. For example, respondents from Denmark noted that evaluation is frequently not considered to be important, while in France provider networks reportedly perceive evaluation, which is mandatory, as a mechanism of 'control' rather than a means to produce evidence to inform service improvement.

The potential need for, or benefit of, conducting evaluations may generally be largely unknown to policymakers and practitioners, as indicated by respondents from Spain, thus acting as an important barrier to implementation of evaluation of programmes. As a consequence of low levels of awareness, capacity to perform evaluation is likely to be low and so will be provider interest in evaluation:

I think we absolutely have a challenge, there is no culture of evaluation. [...] The culture of the organisation [of healthcare] is not very prone to evaluation. [...] If you have never done it, you don't know how to do it, there it is quite difficult to start it and nobody has asked for it in the past (Spain).

The implied lack of capacity to undertake sound evaluation was further highlighted by respondents from Denmark. Concerns were raised particularly in the context of the 2007 administrative reform which introduced a new role for municipalities in the healthcare sector while lacking a coherent framework to guide municipalities in their new tasks. As a consequence:

[W] hat is happening now is that you have 98 municipalities [bidding for transition funds] in 98 different ways, [...] and they are not very good at evaluating what they do because they are so small and evaluation is very expensive. [... This] means that we don't get any knowledge of things that they are doing within the municipalities, so we have a lot of knowledge being wasted (Denmark).
Box 1: Framework for evaluating provider networks in France: national standards and local practice

In France, in 2002, the funding of provider networks was tied to the obligation of conducting a triennial external evaluation [25]. Initially, guidance on evaluation was limited to recommendations issued by the National Authority for Health (Haute Autorité de Santé, HAS) [49]. These recommendations are structured around five themes: (1) network objectives, (2) integration of users and professionals in the network, (3) functioning of the network (4) quality of care and (5) economic evaluation. These recommendations were formalised in 2007 by means of a ministerial circular, which added to the evaluation domains used by the HAS the requirement of pre-post comparison or comparison with 'other initiatives', although these 'other initiatives' were not defined further [50].

Among the 12 provider networks examined in the case study, external evaluations were found to broadly follow the HAS stipulations, although, in practice the evaluation requirements were very rarely met in their entirety. Importantly, only 4 out of the 12 provider networks reviewed did use a comparative design. Furthermore, existing evaluations rarely carried out sound economic assessment beyond budget descriptions.

While it is difficult, on the basis of these observations, to assess cause and effect, findings seem to suggest that national recommendations in France may be too broad and lack sufficiently detailed guidance for those interested in conducting sound evaluation. There is a tension between the ambition, at national level, to standardise and the requirements of existing initiatives which are very diverse and therefore defy a unique framework for evaluation. Importantly however, provider networks tend to have emerged as small bottom-up initiatives, founded and operated by health professionals; as such, networks in France are not equipped to collect the exhaustive data necessary for comparative evaluation. Additionally, external evaluators may not have the skills and financial, as well as human, resources needed for a task as complex as comparative evaluation.

In France, respondents noted that evaluation is a recent phenomenon and therefore there is a lack of experienced evaluators. This was further illustrated by the French case study (Box 1), which highlighted a lack of skills to undertake comparative evaluation among those tasked with the external evaluation of provider networks.

The French case study also identified the lack of guidance as a potential barrier to the more systematic implementation of evaluation, in particular against the background of a perceived or real lack of skill or capacity to carry out evaluation. A similar issue was raised by one key informant from Austria who noted that so-called reform pool projects; that is, projects, funded by the states that seek to better integrate care, have a mandatory commitment to also undertake an evaluation. However, the nature and scope of the required evaluation "is not so very well defined". 
While the existence of a general culture of evaluation, however defined, may be a necessary condition, it might not be sufficient. Respondents from The Netherlands, who highlighted the role of The Netherlands Institute for Health Research and Development (ZonMw) in promoting evaluative research and paving the way for a more evaluative culture, acknowledged the continued need to demonstrate the benefits of evaluation:

[W]ell I don't know whether it's culture already. From my organisation we [...] still have the idea that we have to fight [for] it (The Netherlands).

\subsection{Reluctance of payers and providers to engage in evaluation}

Interview respondents in a number of settings noted that funders and/or providers may be unwilling to provide adequate support to undertake sound evaluation. This may be prompted by a general lack of evaluation culture as noted above, further reinforced by different rationales acting at various levels of the decision-making process:

Policy-makers are [...] not paying attention to change at the micro level, at the level of [health] professionals and we need to make [it] mandatory [...] to evaluate all different programmes (Spain).

Several informants also highlighted a lack of overall interest in wanting to know whether a given innovation does in fact result in improvements in processes or outcomes. This reluctance can be driven by a range of factors. For example in Germany, until recently, statutory health insurance funds, which offer structured disease management to their enrolled population, received additional funding for every patient registered in a disease management programme (DMP) [26]. As a consequence, for those funds with a high membership of patients with chronic disease, there may have been a low interest in identifying whether or not disease management 'works' because of the risk of losing this additional funding. Conversely, for those funds with a high membership of relatively healthy patients, who benefited less from the financial incentive, proof of DMP effectiveness was equally undesirable:

There were clear political reasons. [...] So for some sickness funds it was a very attractive idea to have DMPs because they had more chronic ill patients who are eligible for those programmes. They earned a lot of money [from this system]. [...] Other sickness funds [...] calculated that they would lose a lot of money because they had not so [many registered] chronically ill patients. [...] And those sickness funds tried to stop the programmes on a political basis.[...] This was a big battle. They wanted to stop the connection of those DMPs to the RSA [risk structure compensation scheme], so they had no interest in proof of success of those programmes. And this is a big political issue (Germany).

Other informants from Germany highlighted a historical lack of interest among funders in "what is done with their money" although this has been changing over the years, with an increasing "consensus about the necessity to have evaluation of the effectiveness of money spent by the State or by the sickness funds." However, this has yet to be translated into more systematic financial support for evaluation:

[O]n a small scale there is little but more done than in former years, there is a research programme on the way on health care research but this is small if you compare it to biomedical research on the human genome or the molecular basis. Much more money is going into this basic science stuff than in health care research and evaluation of concrete care. (Germany)

Several informants also pointed to a perceived or real reluctance of providers in supporting evaluation, for example as a result of the additional administrative burden this entails. There may be uncertainties about the range of relevant indicators and the feasibility to collect them, alongside questions, among providers, about the validity of metrics used for evaluation; such metrics were commonly perceived as not necessarily representing the 'true' quality of care provided (see also Box 2). This notion was reported in several countries, with interviewees noting that making available such data (for example, to health insurers) may be interpreted by providers as a compromise on their freedom of practice to the extent that this would likely involve subjecting their practice to more external scrutiny:

[...] some doctors [say] that they don't want to collect data because they are afraid about the 'big brother' syndrome, [...] of being judged on their data. In Denmark there are many small units of GPs: one-third of GPs are alone in their own practice and therefore it is easier to blame them if the data are not good enough (Denmark).

This reluctance of physicians to disclose patient data and outcomes to payers and other stakeholders may also reflect the importance that providers assign to their professional independence. The underlying concern seems to be that if treatment and outcomes are made transparent this might interfere with the doctor-patient relationship and thus impact on patient care.

Against this background, respondents highlighted the need to engage clinicians in the process more actively so as to come to a common understanding about the purpose of the evaluation, and thereby strengthen support, as noted for Germany:

Ambulatory [care] physicians have a lot of work with these DMPs, about the evaluation. They have to give a lot of information but this information is more or less used for regulatory purposes [only] and is not a good feedback for the practices. For example, they don't know what happens to the patient, they have got very bulky reports. [...] They are not very happy that they have to write a lot of data, a lot of work with that, and the results of the evaluation are not quite transparent and useable. (Germany)

Similar points were also highlighted in the Dutch case study, which reported on the views of practitioners involved in evaluation of diabetes care programmes (Box 2 ). While noting the tension between what programme managers and health insurers expect from the evaluation 


\section{Box 2: Attitudes to disease management evalu- ation on the ground: a perspective of practition- ers in The Netherlands}

The Dutch case study aimed to assess health professionals' attitudes towards disease management evaluation [51]. With regard to the determination and assessment of programme goals, respondents agreed that a clear understanding of the benchmarks against which to measure is essential in order to work towards higher quality. Providers expressed a concern that evaluation is perhaps too focused on the 'end product' of care (clinical outcomes), which in their opinion does not reflect the quality of care delivered. Processes and structures are evaluated less structurally and/or solely when outcomes are poor.

Eighteen respondents considered more patientcentred outcomes, especially quality of life, to be important quality indicators, even though they are viewed as difficult to measure and interpret. There appeared to be general consensus $(N=20)$ that parameters focusing on the knowledge and behaviour of patients should form a standard component of measuring diabetes care quality, to allow for more insight into patients' self-management capabilities. Overall, however, respondents disagreed about the optimum 'mix' of indicators suited for quality measurement: while 15 interviewees considered a limited number of mostly process and outcome measures to be sufficient, 12 respondents argued for a wider range of indicators.

There were some concerns about the involvement of government, research organisations and, in particular, health insurers in indicator development. These centred on providers' workload becoming too heavy, which might result in unreliable data registration, and on health insurers' tendency to focus on costs rather than quality. At the same time, the majority of respondents believed that a cooperative approach might be beneficial by allowing for a broader evaluation perspective beyond the traditional biomedical approach. Eighteen respondents reported having experienced difficulties in evaluating the quality of diabetes care. Such difficulties were often of a practical nature and related to shortcomings in IT infrastructure, lack of time for data documentation, and/or limited experience in quality measurement in primary care.

Similar to what was observed for France (Box 1), there appears to be a need in The Netherlands to provide better support to care groups 'on the ground', to enable data collection and analysis in order to support evaluation of intervention effect.

and views of practitioners involved in providing care (and collecting the data for evaluation), practitioners also noted the benefits of a cooperative approach that allows for a broader evaluation perspective.

Also, in France, interviewees reported that professional relationships often have a tendency to be based on the affective and informal. Hence, an implemented evaluation may be biased by "good will" of the evaluator who would have good relations with the financing body and the evaluated structure [27].

\subsection{Data issues and heterogeneity of IT infrastructure}

A common challenge across countries which can restrict the types of evaluation that can be adopted in practice is the available information technology (IT). This challenge appears to exist in different forms: a lack of infrastructure, difficulty of use, and wide varieties of IT systems. Such concerns were reported by a number of respondents, noting that providers often use different documentation systems within and between sectors. Information systems operated by providers are not necessarily compatible and may necessitate adjustment to meet requirements imposed by evaluators, limiting the possibility of pooling data for evaluations. In The Netherlands, survey respondents also noted challenges posed by the use of different clinical information systems across care providers (hospitals, GPs, etc.). Dutch interviewees explained this further:

We have done the bundled payment introduced 3-4 years ago and on an experimental basis, for diabetes only. Some care groups just started to work with this bundled payment and the negotiations, and we have been evaluating ten care groups [using bundle payment, and for diabetes only]. We [...] got registration data from their general practices on what they were doing and this evaluation was finished last year. The process was improving but on outcome we could not see anything, partly because the time period was too short but also because it was very difficult for the care groups to give information from their register to us. They found it difficult to exchange the information on that: [...]a care group consists of about 100 [...] general practitioners [and other allied health professionals]. [...] They all have their own system for registering and what they are doing and what is the outcome of it. And the connection between the different systems is not ready yet. There are care groups who can provide the information but most have many difficulties (The Netherlands).

Denmark has a well-established IT system in healthcare services, where data are systematically collected from family or general practice (GP), stored in a GP database, and submitted to the Danish National Indicator Project (NIP) [28]. The importance of identifying areas of excellence and assessing the outcomes of disease management initatives through the generation of data sets and the NIP was expressed by key informants, explaining that:

[...] then we can follow the patient throughout the different levels of care and [with the data we collect] we can go to the politicians and say 'Look, you must [address chronic disease] another way. But we must have data before we can do it, before we can take [up] discussions. There are so many big feelings in that field, we must have data before we can convince [decision-makers] (Denmark).

Even in Denmark data challenges were reported, insofar as the diverse IT systems that are in place tend to be difficult to use for both data input and output.

In Austria, the use of different documentation systems in inpatient and outpatient care limits the possibilities of using this data for evaluation such as when patient 
follow-up might be required. The inability to link data presents an important challenge in many settings, frequently hindering the use of appropriate evaluation approaches. For example, in Germany, there is good quality basic data available in specific regions and to specific statutory health insurance funds. Yet, it is not possible to create a greater pool of data suited for evaluation because the linking of data across different regions and/or across different funds remains a challenge. This has been attributed to limited interest of policymakers and issues around data confidentiality:

[Because of issues around] data confidentiality, [...] we do not have good studies [which can] compare different kinds of care (Germany).

The use and sharing of data is also reported to be a major barrier in France. In order to allow for the use of statistical controls (e.g. to perform a rigorous economic evaluation), access to comprehensive data held by local health insurance is required. Such access has so far only been granted in very rare cases; the criteria for permitting or refusing access to complete data are not made transparent.

\section{Discussion}

This study contributes to our understanding of some of the most common underlying barriers to chronic care evaluation by highlighting the views and experiences of stakeholders and experts in six European countries. A limitation of our study is that the number and types of stakeholders interviewed varied across countries. We employed various techniques to increase response rates (e.g. offering to undertake interviews in native language; translating the interview guide into Spanish and French) but efforts to capture a large number of views of different stakeholders were sometimes unsuccessful. A particular shortcoming was low participation of patient representatives, in spite of efforts to increase the all-important representation of patients in our study. A second limitation is that we focused on conducting key informant interviews in only six European countries. Drawing on key informants from a larger sample of countries would have yielded a more robust evidence base in relation to our underlying hypothesis.

Evaluations are seldom "value neutral" because stakeholders will have interests to defend; these interests must be taken into consideration to make the evaluation relevant and appropriate [29]. Unlike a performance audit, evaluation seeks to form a 'judgement' about that performance [30]. And, although such judgement is ideally made against a set criteria using a transparent and objective selection of measures, the very prospect of 'being judged' may be reason enough to explain the limited evaluation culture in chronic care, as we found here. Taut and Dieters [31] take a psychological perspective to explain stakeholders' resistance to evaluation, and corroborate many of the expert views presented in this paper. They note that "by definition, evaluation makes performance transparent" and this has relevance to self-image, perception of others, and considerations of power and influence. This links to political science views on evaluation wherein change is implied, contradicting and threatening the interests of existing institutional arrangements [32]. Further, cultural barriers to evaluation may be linked to the degree by which societies embrace the concept and application of accountability. In some European countries, for instance, this concept is still recent (and may even lack a corresponding term); in France, evaluation of public initiatives has long been considered a domain of state elites only [33]. Stakeholders and evaluators may therefore find themselves "captured" by conflicting policy goals, interests and prevailing practice.

This study also highlighted the shortcomings of existing IT systems in supporting chronic care evaluation. Insufficient and/or heterogeneous IT structures hamper the systematic registration of patient data that is necessary for improving and evaluating the quality of chronic care. Already in the mid-1990s, there were concerns about the medical care field being far behind most other industries in using electronic data [34]. This research draws attention to the fact that current clinical information systems in countries reported here still may not entirely meet health professionals' information needs. There is scope for continued efforts at developing approaches to implement electronic health records [35] and studying whether they help to improve the quality and efficiency of chronic care provision [5]. Designing and implementing a commonly accepted workflow system for chronic care, based on universally accepted guidelines (risk management, disease management and case management) could generate consistent data. Moreover, supporting data protection reform across the EU could enable more efficient and safe data sharing [36].

\section{Conclusions}

Arguably a key challenge to evaluation of chronic care initiatives lies in the complexity of interventions, and in the often short-term nature of current service delivery, making it difficult to structurally embed evaluation in daily practices. However methodological and practical challenges to evaluation may be more straightforward to address than the cultural and political barriers to evaluation of chronic care discussed in this paper. Thus it is essential to understand the broader context within which these interventions and their evaluation are designed.

Healthcare professionals should be more engaged in pushing for and supporting measures to evaluate chronic care. They could for example be motivated by using incentives such as regular feedback on their performance [37], which may improve clinical practice behaviour [38]. There may be lessons to be learned from the emergence of evidence-based medicine (EBM) in the 1990s [39,40], which led to efforts in applying scientific rigour in the development of high quality and valid clinical guidelines [41]. Though there is still a great deal of improvement to be done both on the quality [42] and the implementation of clinical guidelines $[43,44]$, this movement has signified a shift in decision-making, moving from a reliance on individual clinical expertise towards an application of empirical "collective" evidence to validate clinical decisions $[45,46]$. Many factors converged to drive the EBM 
movement, especially the realisation that many, if not most, daily clinical decisions were not based on valid scientific fact [47], an acknowledgement of upward pressure on healthcare costs and therefore pressure to make the best use of limited resources, and a public more engaged in treatment options [48].

The infrastructure and culture for evaluation must however be driven by decision-makers and those funding chronic care initiatives. Incremental behaviour change can be facilitated by creating an appropriate framework for evaluation. For instance, it should be ensured that evaluators receive independent funding and do not have institutional proximity to the evaluated structure. Payers need the right incentives to promote and improve programme evaluation.

There is a need to find a balance between the importance of a given intervention and the value of the evidence that can be collected against the background of constraints [12], be they financial, cultural or otherwise. While this will be important in any context, such trade-offs are likely to pose a particular challenge in those settings where the 'evaluation culture' is weak or of low priority.

\section{Acknowledgments}

The DISMEVAL project was funded under the European Commission's Seventh Framework Programme (FP7) (grant no. 223277). The views expressed in this paper are those of the authors alone and the European Commission is therefore not liable for any use that may be made of the information contained herein.

We would like to express our gratitude to interview participants for kindly giving their time and sharing their knowledge.

\section{References}

[1] Nolte E, Knai C, McKee M. Managing chronic conditions: experience in eight countries. Copenhagen: World Health Organization on behalf of the European Observatory on Health Systems and Policies; 2008.

[2] de Bruin SR, Heijink R, Lemmens LC, Struijs JN, Baan CA. Impact of disease management programs on healthcare expenditures for patients with diabetes, depression, heart failure or chronic obstructive pulmonary disease: a systematic review of the literature. Health Policy 2011;101:105-21.

[3] Gress S, Baan CA, Calnan M, Dedeu T, Groenewegen P, Howson H, et al. Co-ordination and management of chronic conditions in Europe: the role of primary care - position paper of the European Forum for Primary Care. Quality in Primary Care 2009;17:75-86.

[4] Nuno-Solinis R, Orueta JF, Mateos M. An answer to chronicity in the Basque Country: primary care-based population health management. Journal of Ambulatory Care Management 2012;35:167-73.

[5] Nolte E, McKee M. Caring for people with chronic conditions. A health system perspective. Copenhagen: World Health Organization on behalf of the European Observatory on Health Systems and Policies; 2008.

[6] McKee M, Nolte E. Responding to the challenge of chronic diseases: ideas from Europe. Clinical Medicine 2004;4:336-42.

[7] Nolte E, Conklin A, Adams A, Brunn M, Cadier B, Chevreul K, et al. Evaluating chronic disease management. Recommendations for funders and users. DISMEVAL Project. Prepared for the European Commission on behalf of the DISMEVAL Consortium; 2012

[8] Linden A, Adams JL, Roberts N. Strengthening the case for disease management effectiveness: un-hiding the hidden bias. Journal of Evaluation in Clinical Practice 2006;12:140-7.

[9] Linden A, Roberts N. A user's guide to the disease management literature: recommendations for reporting and assessing program outcomes. American Journal of Managed Care 2005;11:113-20.
[10] Steuten L, Vrijhoef B, Severens H, van Merode F, Spreeuwenberg C. Are we measuring what matters in health technology assessment of disease management? Systematic literature review. International Journal of Technology Assessment in Health Care 2006;22:47-57.

[11] Campbell NC, Murray E, Darbyshire J, Emery J, Farmer A, Griffiths F, et al. Designing and evaluating complex interventions to improve health care. BMJ 2007;334:455-9.

[12] Craig P, Dieppe P, Macintyre S, Michie S, Nazareth I, Petticrew M. Developing and evaluating complex interventions: new guidance. London: Medical Research Council; 2008.

[13] Ogilvie D, Cummins S, Petticrew M, White M, Jones A, Wheeler K. Assessing the evaluability of complex public health interventions: five questions for researchers, funders, and policymakers. Milbank Quarterly 2011;89:206-25.

[14] Cretin S, Shortell SM, Keeler EB. An evaluation of collaborative interventions to improve chronic illness care. Framework and study design. Evaluation Review 2004;28:28-51.

[15] Linden A, Adams JL, Roberts N. Evaluating disease management program effectiveness: an introduction to time-series analysis. Disease Management 2003;6:243-55.

[16] The DISMEVAL Project Consortium. Developing and validating disease management evaluation methods for European healthcare systems. Final report, DISMEVAL Project. Prepared by the Project Coordinator for the European Commission on behalf of the DISMEVAL Consortium; 2011.

[17] Gilson L, Raphaely N. The terrain of health policy analysis in low and middle income countries: a review of published literature 1994-2007. Health Policy and Planning 2008;23:294-307.

[18] Kingdon J. Agendas, alternatives, and public policies. New York: Longman; 1995.

[19] Reich M. Political mapping of health policy - a guide for managing the political dimensions of health policy. Boston, MA: Harvard School of Public Health; 1994.

[20] Walt G. Health policy: an introduction to process and power. London: Zed Books; 1994

[21] Walt G, Gilson L. Reforming the health sector in developing countries: the central role of policy analysis. Health Policy and Planning 1994;9:353-70.

[22] Walt G, Shiffman J, Schneider H, Murray SF, Brugha R, Gilson L. 'Doing' health policy analysis: methodological and conceptual reflections and challenges. Health Policy and Planning 2008;23:308-17.

[23] Leichter H. A comparative approach to policy analysis: health care policy in four nations. Cambridge: Cambridge University Press; 1979.

[24] Ritchie J, Spencer L. Qualitative data analysis for applied policy research. In: Bryman A, Burgess R, editors. Analysing qualitative data. London: Routledge; 1994.

[25] Chevreul K, Brunn M, Cadier B, Durand-Zaleski I, Farsi F, Labrosse H. Evaluation of disease management in France. In: Nolte E, Hinrichs S, editors. Developing and validating disease management evaluation methods for European healthcare systems Final report. Santa Monica, CA: RAND Corporation and DISMEVAL Consortium; 2012.

[26] Nolte E, Knai C, Hofmarcher M, Conklin A, Erler A, Elissen A, et al. Overcoming fragmentation in health care: chronic care in Austria, Germany and The Netherlands. Health Economics, Policy, and Law 2012;7:125-46.

[27] Noguès F, Noguès E. Évaluation des réseaux de santé en France. Risques and Qualité en Milieu de Soins 2009;3:139-44

[28] Grosen L. Electronic Health Record in Denmark. Health Policy Monitor; October 2009.

[29] Swanson B, Bentz R, Sofranko A. Improving agricultural extension. A reference manual. Rome: Food and Agriculture Organization of the United Nations; 1997.

[30] Ling T. A framework for understanding the contribution of public services to public benefit. In: Ling T, Villalba van Dijk L, editors. Performance audit handbook: routes to effective evaluation. Cambridge, UK: RAND Europe; 2009.

[31] Taut S, Brauns D. Resistance to evaluation. A psychological perspective. Evaluation 2003;9:247-64.

[32] Wildavsky A. The self-evaluating organization. Public Administration Review 1972;32:509-20.

[33] Barbier J. Eléments pour une sociologie des évaluations publiques en France. Revue Française des Affaires Sociales 2010;64:27-50.

[34] Shortell S, Gillies R, Anderson D, Erickson K, Mitchell J. Remaking health care in America: building organized delivery systems. San Francisco: Jossey-Bass; 1996.

[35] Burton LC, Anderson GF, Kues IW. Using electronic health records to help coordinate care. Milbank Quarterly 2004;82:457-81.

[36] European Commission. Commission proposes a comprehensive reform of the data protection rules (http://ec.europa.eu/ justice/newsroom/data-protection/news/120125_en.htm); 2012. 
[37] Chevreul K, Le Fur P, Sermet C. Systèmes d'information sur les pratiques médicales de ville: enseignements à partir de quelques expériences étrangères. Questions d'économie de la santé, Irdes, $2004 ; 85$.

[38] Frenzel JC, Kee SS, Ensor JE, Riedel BJ, Ruiz JR. Ongoing provision of individual clinician performance data improves practice behavior. Anesthesia and Analgesia 2010;111:515-9.

[39] Guyatt G. Evidence-based medicine. ACP Journal Club 1991;114:A16.

[40] Evidence-Based Medicine Working Group. Evidence-based medicine. A new approach to teaching the practice of medicine. JAMA 1992;268:2420-5.

[41] Burgers JS, Grol R, Klazinga NS, Makela M, Zaat J. Towards evidencebased clinical practice: an international survey of 18 clinical guideline programs. International Journal for Quality in Health Care 2003; 15:31-45.

[42] Knai C, Brusamento S, Legido-Quigley H, Saliba V, Panteli D, Turk $\mathrm{E}$, et al. Systematic review of the methodological quality of clinical guideline development for the management of chronic disease in Europe. Health Policy 2012;107(2):157-67.

[43] Brusamento S, Legido-Quigley H, Panteli D, Turk E, Knai C, Saliba V, et al. Assessing the effectiveness of strategies to implement clinical guidelines for the management of chronic diseases at primary care level in EU Member States: a systematic review. Health Policy 2012;107(2):168-83.

[44] Legido-Quigley H, Panteli D, Brusamento S, Knai C, Saliba V, Turk E, Solé M, Augustin U, Car J, McKee M, Busse R. Clinical guidelines in the
European Union: Mapping the regulatory basis, development, quality control, implementation and evaluation across member states. Health Policy 2012;107(2):146-56.

[45] Degen RM, Hodgins JL, Bhandari M. The language of evidence based medicine: answers to common questions? Indian Journal of Orthopaedics 2008;42:111-7.

[46] Mayer D. Evidence-based medicine. Epilepsia 2006;47(Suppl.), $1: 3-5$.

[47] McDonald CJ. Medical heuristics: the silent adjudicators of clinical practice. Annals of Internal Medicine 1996;124:56-62.

[48] McQueen MJ. Overview of evidence-based medicine: challenges for evidence-based laboratory medicine. Clinical Chemistry 2001;47:1536-46.

[49] ANAES (HAS). Evaluation des réseaux de soins: bilan de l'existant et cadre méthodologique. Paris, former Agence Nationale d'Accréditation et d'Evaluation en Santé (ANAES), now part of the Haute Autorité de Santé (HAS); 2001.

[50] Ministry of Health F. Circulaire DHOS/O3/CNAM n ${ }^{\circ}$ 2007-88 du mars 2007 relative aux orientations de la DHOS et de la CNAMTS en matière de réseaux de santé et à destination des ARH et des URCAM; 2007.

[51] Elissen A, Duimel-Peeters I, Spreeuwenberg C, Vrijhoef H. Evaluation of disease management in The Netherlands. In: Nolte E, Hinrichs S, editors. Developing and validating disease management evaluation methods for European healthcare systems Final report. Santa Monica, CA: RAND Corporation and DISMEVAL Consortium; 2012. 